Aus Dem Afatom. Ingtitut der Univeritía Utrecht (Holland).

\title{
EINE VARIATION IM BEREICH \\ DER VENA CAVA INFERIOR UND DER VENAE CARDINALES POSTERIORES.
}

\author{
VON \\ H. B. GOETTSCH, \\ CAND. MED.
}

Mit 13 Abbildungen im T'ext. 

Im Präpariersaal der Utrechter Anatomie wurde im Wintersemester 1916-17 in der Leiche eines erwachsenen Mannes eine Abweichung des Rumpfvenensystems angetroffen.

Im folgenden soll zunächst der Befund beschrieben werden, danach ist die Deutung. desselben mit Bezug auf andere ähnliche Beobachtungen zu besprechen.

\section{Beschreibung des Befundes.}

Die abführende Bahn des Blutes der linken unteren Extremität und der linken Beckenhälfte steigt an der linken Seite der Lendenwirbelsäule empor. Sie liegt dabei dorsal von deŕ Art. iliaca communis sinistra.

Der Stamm, welcher das Blut der rechten unteren Extremität centralwärts führt, liegt erst rechts von, dann vor der Lendenwirbelsäule. Auf der Höhe dès dritten Lendenwirbels vereinigt er sich mit dem linksseitigen Stamm. Während seines Verlaufes vor der Lendenwirbelsäule kreuzt er die Aorta an dessen dorsaler Seite.

Bevor die Vereinigung zustandekommt, nimmt die linke Extremitätenvene drei Zweige auf, wovon einer an der lateralen Seite zur Höhe des untersten Lendenwirbels einmündet, während zwei an der dorso-medialen Seite einmünden. Der oberste dieser letzteren Zweige, welcher seinen Verlauf entsprechend als Lumbalvene zu bezeichnen ist, verbindet sich mittels einer Anastomose mit der Vereinigungsstelle der beiden Extremitätenstämme (Fig. 2). 


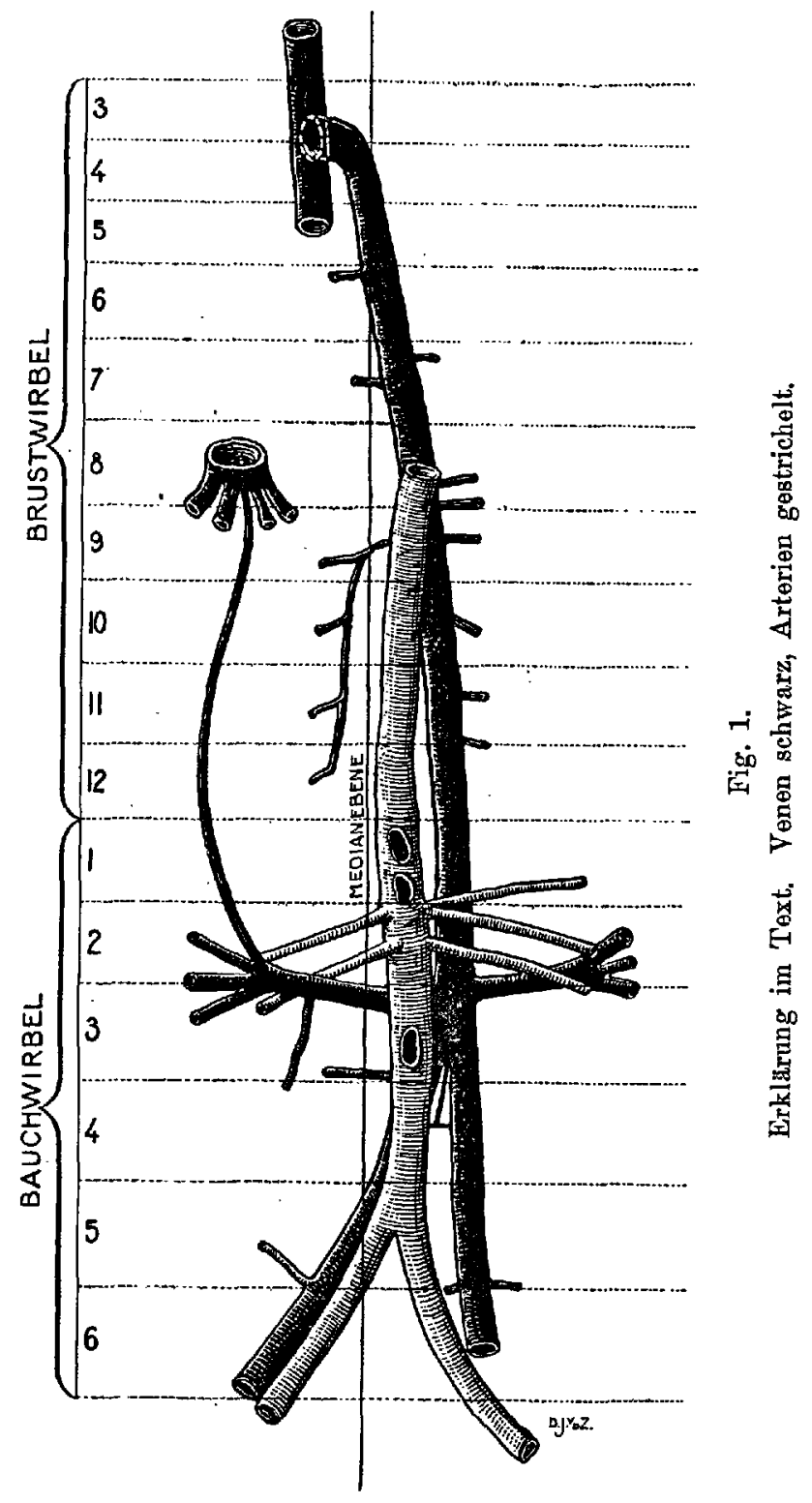


Die rechte Extremitätenvene nimmt während ihres Verlaufes vor der Wirbelsäule zwei als Lumbalvenen zu bezeichnende Seitenzweige auf. Eine Längsanastomose zwischen Lumbalvenen fehlt beiderseits; es wurden keine Venae lumbales ascendentes angetroffen.

Beiläufig sei erwähnt, dass die Wirbelsäule des betreffenden Individuums zwölf Brust- und sechs Bauchwirbel hat.

Der aus der Vereinigung der beiden erwähnten Extremitätengefässe hervorgehende Stamm bleibt weiter cranial vorläufig

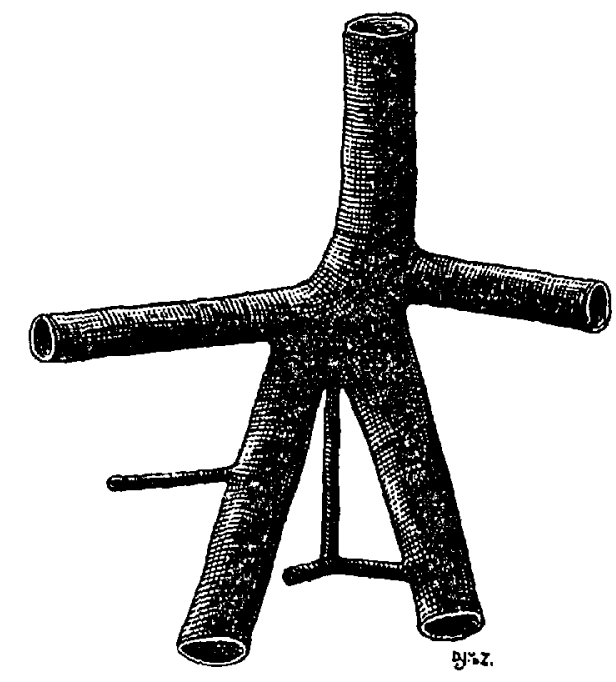

Fig. 2.

Vereinigungsstelle der Extremitatenvenen.

an der linken Seite der Wirbelsüule (s. Fig. 1). Er nimmt sofort nach der Vereinigung eine von der rechten Niere kommende Vene auf, welche, hinter der Aorta verlaufend, den Hauptstamm erreicht zur Höhe der Zwischenwirbelscheibe L 2-L 3.

Etwas höher mündet die von der linken Niere kommende Vene ein.

Uber die Einmündungsverhältnisse der Venae spermaticae fehlen leider genaue Daten. Die linke Vene wurde nicht auf- 
gefunden, die rechte vereinigt sich mit dem von der rechten Niere kommenden Stamme.

Der Hauptvenenstamm liegt in seinem Verlauf weiter cranialwärts zunächst dorsal von drei Arterien, welche, von der Aorta kommend, sich zur linken Niere und Nebenniere begeben. Die rechte Niere empfängt zwei Arterien.

Gemeinschaftlich mit der Aorta erreicht nun der Hauptstamm die Brusthöhle. Hier verlässt das Gefäss' allmählich die linke Seite der Wirbelsäule, die Aorta dorsal überkreuzend, überschreitet die Medianlinie und vereinigt sich zur Höhe des vierten Brustwirbels mit der oberen Hohlvene.

In den thoracalen Abschnitt. münden linkerseits sieben segmentale Venen ein; rechterseits münden vier derartige Gefässe, welche durch eine Längsanastomose verbunden sind, gemeinschaftlich ein, während zwei, weiter cranialwärts, gesondert sich mit dem Hauptstamm vereinigen.

Ein kleines Gefäss, welches die rechte Nierenvene mit der Vena hepatica revehens communis verbindet, verdient besondere Beachtung. Von der Stelle aus, wo das Blut die Leber verlässt, wurde nach einer etwa vorhandenen unteren Hohlvene gesucht; hierbei wurde der kleine eben erwähnte Stamm gefunden, welcher, dorsal von der Leber verlaufend, eine Verbindung zwischen Rumpfwand- und Visceralsystem herstellt.

\section{Verwertung des Befundes.}

Was die Deutung des vorliegenden. Falles anbelangt, so ist zunächst an der Hand einiger schematischer Figuren an die normale Entwickelungsgeschichte des Venensystems $\mathrm{zu}$ erinnern. Zu diesem Zweck wurde versucht, ein Schema zu entwerfen, welches als Grundlage für die Ableitung des normalen Zustandes sowohl als für die zahlreichen in diesem Gebiete anzutreffenden abweichenden Zustände dienen könnte.

In Fig. 3 sind die beiderseits in den Sinus venosus ein- 
mündenden Ductus Cuvieri angegeben. Sie entstehen aus der Vereinigung von vorderen und hinteren Cardinalvenen. Die hinteren Cardinalvenen hängen miteinander durch Anastomosen zusammen, und zwar gibt es zwei Arten von Verbindungen. Erstens werden direkte Anastomosen angetroffen, welche dadurch charakterisiert sind, dass sie dorsal von der Aorta ver-

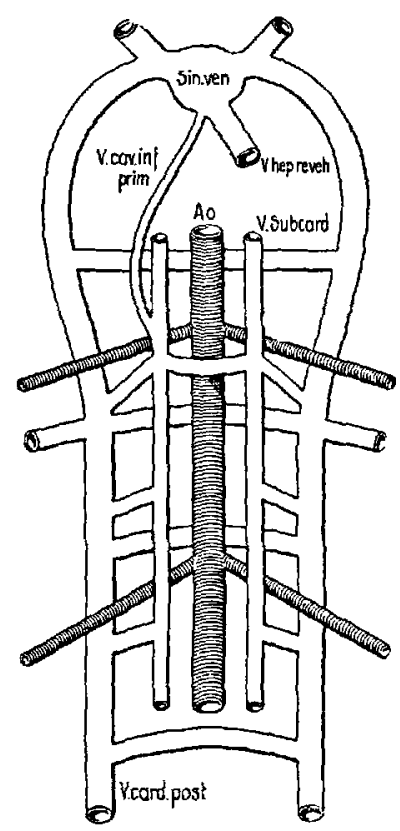

Fig. 3.

Grundplan zur Entwicklung des Venensystems.

laufen. Zweitens kommt eine Verbindung durch Vermittlung der Venae subcardinales zustande, indem diese beiden längsverlaufenden Gefässe sich untereinander unterhalb der Art. mesenterica superior, ausserdem aber durch mehrere Zweige mit den Cardinalvenen verbinden. Das Kennzeichnende der letzteren Anastomose ist dadurch bedingt, dass dieselbe ventral von der Aorta verläuft. Diese beiden Arten von Cardinalanasto- 
mosen sind in der Fig. 3 zu erkennen durch ihr Verhalten zur mitangegebenen Aorta und deren Zweige. Zu beachten ist der Verlauf der letzteren dorsal von den Venae subcardinales und ventral von den Venae cardinales.

Utbersichtlicher sind diese Verhältnisse zwischen arteriellem und venösem System auf dem Querschnitt zu erkennen (Fig. 4). Gestrichelt sind angegeben die dorsal von der Aorta verlaufenden direkten Verbindungen (a), sowie die an der ventralen Seite vorzufindende Anastomose mittels des Subcardinalissystems (b).

Indem im Verlauf der Ontogenese ein capillares Gefäss des rechten Nebengekröses sich zu einem ansehnlichen Stamm er-

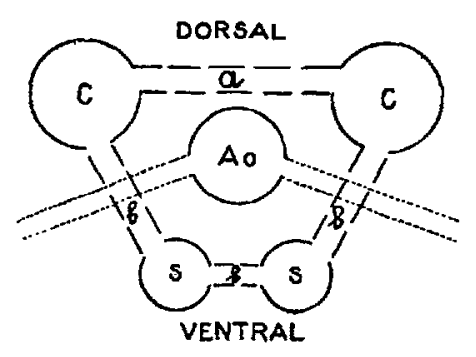

Fig. 4.

Erklärung im Text.

weitert, entsteht die als Vena cava inferior primitiva bekannte Verbindung zwischen der Vena subcardinalis dextra und die Vena revehens hepatis communis. Das Cardinalisblut macht von dieser neuen Bahn Gebrauch, indem es auf kürzerem Wege durch die vorhandenen Anastomosen zwischen den Venae cardinales und subcardinales dem Sinus venosus zufliesst. Die so entstandene Gefüssbahn, welche jetzt das untere Cardinalissystem mit dem Sinus verbindet, entspricht der definitiven Vena cava inferior.

Diese setzt sich als ursprünglich rechte Vena cardinalis posterior distalwärts fort und nimmt dabei durch eine ventrale 'Anastomose (s. Fig. 4) das Blut aus der linken Niere, durch' 
eine dorsale Anastomose (Vena iliaca transversa) das Blut der linken unteren Extremität auf.

Die linke Vena cardinalis posterior ist somit überflüssig geworden, sie obliteriert zum grössten Teil. Nur der proximale Teil bleibt als Vena hemiazygos und hemiazygos accessoria erhalten. Durch eine oder mehrere Verbindungen, welche quer

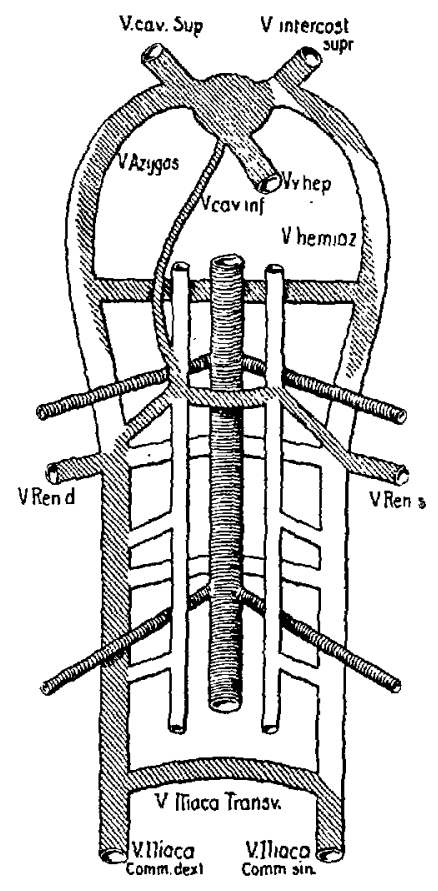

Fig. 5.

Normaler Endzustand, eingetragen im Grundplan.

vor der Brustwirbelsäule hinter der Aorta verlaufen, hängt dieses Gefäss mit dem ebenfalls erhaltenen proximalen Endstücke der Vena cardinalis inferior dextra zusammen. Damit ist der definitive Zustand erreicht, wie ihn Fig. 5 darstellt.

Das Wesentliche der Umbildung, welche das Venensystem der unteren Körperhälfte erfährt, ist, wie aus obigem hervor- 
geht, darin zu suchen, dass der Abfluss des venösen Blutes der unteren Körperhälfte, welcher anfänglich von der oberen Hohlvene (den oberen Hohlvenen) besorgt wird, später durch eine neue Bahn geleitet wird, welche auf kürzerem Wege das Herz erreicht. Die neue Bahn stellt eine Verbindung zwischen dem Cardinalissystem und dem Dotterplacentarsystem dar.

Die Skizze von der Entwickelung des Venensystems, wie sie oben gegeben wurde, ist in manchen Punkten anfechtbar. So bestehen beispielsweise über die Ableitung der Venae azygos und hemiazygos Meinungsverschiedenheiten. Die $\mathrm{Hoch}$ stettersche Auffassung ${ }^{1}$ ), dass wir es hier wenigstens der Hauptsache nach mit den Cardinalvenen zu tun hätten, wird von Parker und Tozier ${ }^{2}$ ), Zumstein ${ }^{3}$ ) und Flor. R. $\mathrm{Sab}$ in ${ }^{4}$ ) bestritten, welche behaupten, dass diese Gefässe als Neubildungen entstehen.

Ähnliches gilt weiter distal für die linke Vena lumbalis ascendens. Während $\mathrm{Lew}_{\text {is }}{ }^{5}$ ) in derselben einen Teil der linken Vena cardinalis posterior sieht, hält Hochstetter ${ }^{6}$ ) diese Ansicht für unrichtig und schreibt die Entstehung dieser Vene einer Kette von sekundären Anastomosen zu.

Es ist nicht meine Absicht, hier auf diese und ähnliche Streitpunkte einzugehen, da mir genügendes embryologisches Material zur Bildung eines eigenen Urteils fehlt.

Auf die dorsal und ventral von der Aorta vorkommenden venösen Anastomosen ist hier noch einzugehen. Die dorsalen direkten Cardinalisverbindungen sind als die primitiveren anzusehen; sie sind den oft zahlreichen Anastomosen zwischen Azygos und Hemiazygos vergleichbar. $\mathrm{Ob}$ sie ursprünglich an der ganzen Wirbelsäule segmental vorhanden sind, wie Fro-
1) 12 , S. 607 .
2) 20 .
3) 27 , S. 188 .
4) 23, S. 84 .
5) 18 , S. 239 .
6) 12, S. 608 . 
ri e $\mathrm{p}^{1}$ ) vermutet, bleibe dahingestellt. Ihr Vorhandensein wird auf Grund eigener Beobachtung von Johnston'), Neuberger ${ }^{3}$ ) und Zumstein ${ }^{4}$ ) bestätigt. Neuberger bemerkt, dass die Vena iliaca transversa nicht als eine dorsale Cardinalisanastomose aufzufassen ist, da sie ventral von der

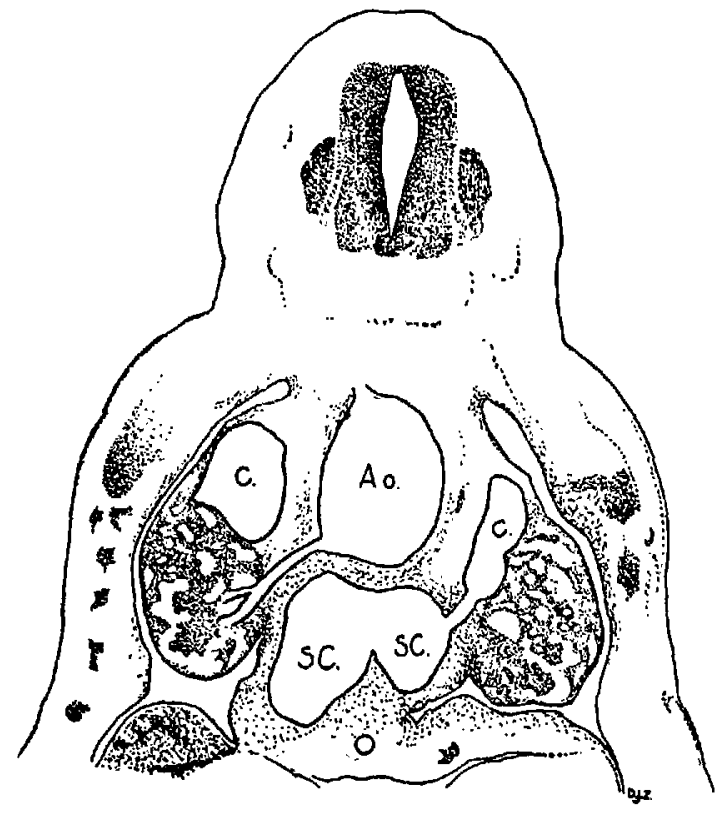

Fig. 6.

Querschnitt durch einem Embryo des Frettchens nach Johnston (14).

Aortafortsetzung (Art. sacralis media) verlaufe. Ich habe auf Grund dieser Bemerkung im Grundplane (Fig. 3) die Aorta nicht bis zur caudalen Cardinalisanastomose eingezeichnet. Diese

1) 4 , S. 581 .

2) 14, S. 238. Er schreibt: "The persistent cross-communications between the two posterior cardinals - - are all post-aortic".

$\left.{ }^{8}\right)$ "Alle sonstige Verbindungen der beiden Kardinalvenen ziehen gemass ihrer topographischen Beziehung dorsal von der Aorta von der einen zur anderen Seite ${ }^{a}(19, S .75)$.

) 26, Taf. XXXIII, Fig. 9 a. 
unterste Verbindung (V. iliaca transversa) wäre als eine Anastomose zwischen den beiden Extremitätenvenen zu deuten.

Die ventrale indirekte Cardinalisanastomose ist aus drei Abschnitten aufgebaut. Zwei symmetrische laterale Teile sind als erweiterte Abschnitte des venösen Wundernetzes der Urniere aufzufassen. Dazwischen liegt die ventral von der Aorta verlaufende Verbindung der beiden Venae subcardinales. Eine Abbildung dieser ventralen Verbindung beim Frettchen, der Arbeit Johnstons ${ }^{1}$ ) entnommen, möge hier Platz finden (Fig. 6). Auch Lew i s ${ }^{2}$ ) bildet dieselbe für das Kaninchen ab. Es ist anzunehmen, dass beim menschlichen Embryo die Verhältnisse ähnlich liegen. Für die zahlreichen Anastomosen zwischen Venae cardinales und subcardinales sei auf die Arbeit Gladstones ${ }^{3}$ ) verwiesen.

Wir kommen jetzt zur Besprechung der abweichenden $\mathrm{Zu}$ stände, wie sie verhältnismässig häufig im besprochenen Venengebiete angetroffen werden. Das oben erläuterte Grundschema soll dabei behilflich sein.

Allgemein kann gesagt werden, dass die meisten betreffenden Variationen als das Erhaltenbleiben primitiver Zustände aufgefasst werden können, indem die Verschiebung des Blutabflusses vom oberen zum 'unteren Hohlvenenstamm vollständig oder teilweise unterbleibt. Es lassen sich diesbezüglich alle Ubergangszustände denken, und eine Einteilung, wie sie im folgenden vorgenommien werden soll, ist demnach als eine künstliche aufzufassen.

Eine naturgemässe Gruppierung hat, wie aus obigem hervorgeht, auf der Art des Abflusses des venösen Blutes entweder durch die primitivere obere oder durch die später auftretende untere Bahn zu beruhen. Diese beiden Abflusssysteme haben sich in jedem Falle das Gleichgewicht zu halten.

1) 14, Fig. 2, S. 239.

7) 18, Fig. 6, S. 231 .

3) 9, Diagramm A, S. 230. 
In einer Gruppe A sinid diejenigen Fälle unterzubringen, bei welchen die neve Bahn überhaupt nicht zur Anlage kommt und das Cardinalissystem, wie beim Embryo, das Blut allein zum Herzen führt ${ }^{1}$ ).

In Gruppe $B$ ist dile Viena cava inferior primitiva zwar vorhanden, aber praktisch spielt sie noch keine Rolle; das Blut erreicht das Herz im wesentlichen durch das persistierende Cardinalissystem.

Gruppe $C$ zeigt das umgekehrte Verhalten. Die Vena cava inferior primitiva hat an Bedeutung gewonnen, der Cardinalisabfluss tritt zurück.

In Gruppe D endlich erreicht das Blut der unteren Körperhälfte ausschliesslich durch die Vena cava inferior primitiva das Herz; die Cardinalvenen führen dem Herzen kein Bauchhöhlenblut zu. An dem Aufbau des unteren Hohlvenensystems können trotzdem auch hier Teile beider hinteren Cardinalvenen beteiligt sein.

Die Art, auf welche in jeder dieser Gruppen das Cardinalissystem erhalten bleibt, bietet theoretisch vier verschiedene Möglichkeiten:

1. Es können beide Cardinalvenen in gleichem Grad erhalten sein.

2. Die rechte Cardinalvene ist ${ }^{\circ}$ als Hauptstamm erhalten, die linke verbindet sich in wechselnder Höhe mit der rechten.

3. Die linke Cardinalvene ist als Hauptstamm erhalten, die rechte verbindet sich in wechselnder Höhe mit der linken.

4. Alternierend treten Bestandteile der rechten und der linken Cardinalvene als Hauptstamm auf.

Schliesslich ist bei den hier zu besprechenden Variationen noch die Lage der rechten oder linken Vena renalis zur Aorta

1) Dwight hat einen zu dieser Gruppe gehörigen Fall beschrieben und die Literatur der ganzen Gruppe zusammengestellt. Literaturliste Nr. 2. 
von Wichtigkeit. Dieses Gefäss kann sowohl ventral wie dorsal von der Aorta verlaufen.

\begin{tabular}{|c|c|c|c|c|}
\hline $\begin{array}{c}\text { Die Vena cava } \\
\text { inferior } \\
\text { primitiva }\end{array}$ & & $\begin{array}{l}\text { Das Cardinalis- } \\
\text { system befordert } \\
\text { zum Herzen }\end{array}$ & $\begin{array}{l}\text { Persisten\%, } \\
\text { Cardinalis- } \\
\text { system }\end{array}$ & $\begin{array}{l}\text { a Vena renalis ven- } \\
\text { tral von der Aorta } \\
\text { b Vena renalis dor- } \\
\text { sal von der Aorta }\end{array}$ \\
\hline fehlt & A & $\begin{array}{c}\text { alles Blut der } \\
\text { unteren Körper- } \\
\text { halfte }\end{array}$ & $\begin{array}{lr}1 & \mathrm{r}+1 \\
2 & \mathbf{r} \\
3 & 1 \\
4 & \mathrm{r} \times 1\end{array}$ & $\begin{array}{l}\mathrm{a} \\
\mathrm{b} \\
\mathrm{a} \\
\mathrm{b} \\
\mathrm{a} \\
\mathrm{b} \\
\mathrm{a} \\
\mathrm{b}\end{array}$ \\
\hline $\begin{array}{l}\text { ist schwach } \\
\text { entwickelt }\end{array}$ & B & $\begin{array}{c}\text { viel Blut der } \\
\text { unteren Körper- } \\
\text { halfte }\end{array}$ & 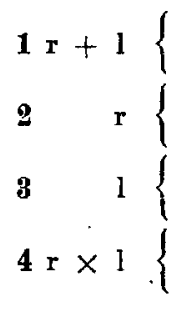 & $\begin{array}{l}\mathbf{a} \\
\mathbf{b} \\
\mathbf{a} \\
\mathbf{b} \\
\mathbf{a} \\
\mathbf{b} \\
\mathbf{a} \\
\mathrm{b}\end{array}$ \\
\hline $\begin{array}{l}\text { ist Haupt- } \\
\text { abflussbahn }\end{array}$ & C & $\begin{array}{c}\text { wenig Blut der } \\
\text { unteren Körper- } \\
\text { hallfte }\end{array}$ & $\begin{array}{l}\mathbf{1}+1 \\
\mathbf{2} \\
\mathbf{3} \\
\mathbf{4} \times \mathbf{1}\end{array}$ & $\begin{array}{l}\mathbf{a} \\
\mathbf{b} \\
\mathbf{a} \\
\mathbf{b} \\
\mathbf{a} \\
\mathrm{b} \\
\mathrm{a} \\
\mathrm{b}\end{array}$ \\
\hline $\begin{array}{l}\text { ist einzige } \\
\text { Abflussbahn }\end{array}$ & $D$ & $\begin{array}{c}\text { kein Blut der } \\
\text { unteren Körper- } \\
\text { halfte }\end{array}$ & $\begin{array}{lr}\mathbf{1} & \mathbf{r}+1 \\
\mathbf{2} & \mathbf{r} \\
\mathbf{3} & 1 \\
\mathbf{4} & \mathbf{r} \times 1\end{array}$ & $\begin{array}{l}a \\
b \\
a \\
b \\
a \\
b \\
a \\
b\end{array}$ \\
\hline
\end{tabular}


In untenstehender Tabelle ist versucht worden, die verschiedenen Variationsmöglichkeiten zusammenzustellen. Nach obigen Auseinandersetzungen wird sie keiner näheren Erklärung bedürfen.

Von den theoretisch möglich zu erachtenden Fällen sind in der Literatur eine Anzahl als tatsächliche Befunde beschrieben worden. Einige derselben mögen hier angeführt werden, sie sind vielfach nicht genügend in Einzelheiten dargestellt, um eine genaue Einordnung möglich zu machen. Besonders verdient erwähnt zu werden, dass diejenigen Fälle, wobei die Vena cava inferior primitiva schwach entwickelt ist, wahrscheinlich oft beurteilt worden sind als zur Gruppe A gehörig, weil der betreffende dünne Stamm, welcher diese Vene darstellt, übersehen worden ist. Mit Recht hebt Neuberger ${ }^{1}$ ) die Anregung $\mathrm{Hochstetters} \mathrm{hervor,} \mathrm{welcher}$ empfiehlt, in allen diesen Fällen die dorsale Wand des Foramen Winslowi genau zu untersuchen.

Gruppe A. Fehlende Vena cava inferior primitiva. Das Cardinalissystem befördert alles Blut der unteren Körperhälfte zum Herzen.

A. 1. Vertreter dieser Gruppe sind $u$. a. beschrieben worden von $\mathrm{HyrtI}^{2}$ ), $\left.\mathrm{Martin}^{3}\right), \quad \mathrm{Dwight}{ }^{4}$ ), H ochstetter ${ }^{5}$ ), Neuberger ${ }^{6}$ ) und Kollmann ${ }^{7}$ ). Der primitivste Zustand, wobei beide Cardinalvenen in ihrer ganzen Ausdehnung $\left(A_{1}\right)$ erhalten sind, ist, in unserem Grundplan eingetragen, als Fig. 7 dargestellt. Ihm entsprechen die Fälle von $\mathrm{H}$ y rtl und $\mathrm{M}$ a r tinn.

A.2. Fälle, wo nur die rechte Cardinalvene in ihrer ganzen Ausdehnung erhalten blieb, sind in grosser Anzahl von $\mathrm{D}$ w i g h t

\footnotetext{
1) 19, S. 76 .

2) Siehe 4 .

3) Siehe 4.

4) 2.

s) 12 .

6) 19 .

7) 15 .
} 
zusammengestellt worden. Es sei diesbezüglich auf seine Arbeit verwiesen. Von den zahlreichen verwirklichten Möglichkeiten, zahlreich, weil das Verhalten der linken Cardinalvene

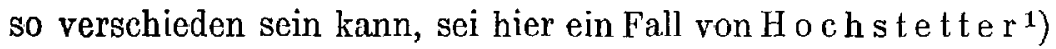
herausgegriffen (Fig. 8). Der Fall ist dadurch charakterisiert, dass die linke Nierenvene nicht unmittelbar mit der rechten Cardinal-

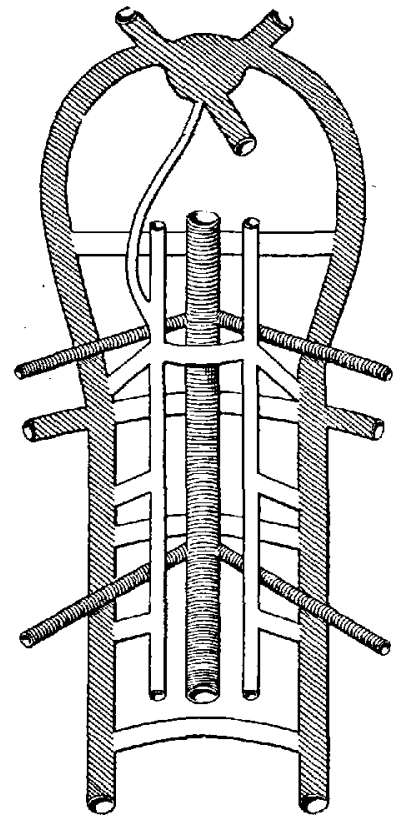

Fig. 7.

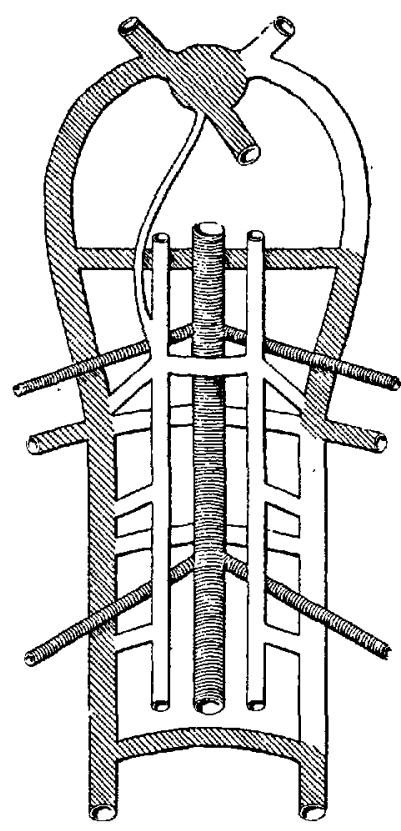

Fig. 8.

Variation A 2. Fall von Hoch. stetter.

vene zusammenhängt, sondern mittels eines Teils der linken Cardinalvene, welche erst im Thoraxgebiet (9. Brustwirbel) hinter der Aorta mit der Hauptabflussbahn zusammenhängt.

A.3. Beispiele vom Erhaltenbleiben der linken Cardinalvene sind in Dwights Zusammenstellung ebenfalls anzu-

1) 12, S. 631, Fig. 14. 
treffen. Ein später veröffentlichter Fall ist der von $\mathrm{N} \in \mathbf{u}$ b e rge $\mathrm{r}^{\mathrm{y}}$ ), welcher hier, im Grundschema eingetragen, Platz finden möge. Es handelt sich um einen Befund, wobei die Vena renalis der rechten Seite, vor der Aorta verlaufend, den linken Cardinalisstamm erreicht (A.3. a.). Von der rechten Cardinalvene ist hier überhaupt nichts erhalten geblieben, es sei

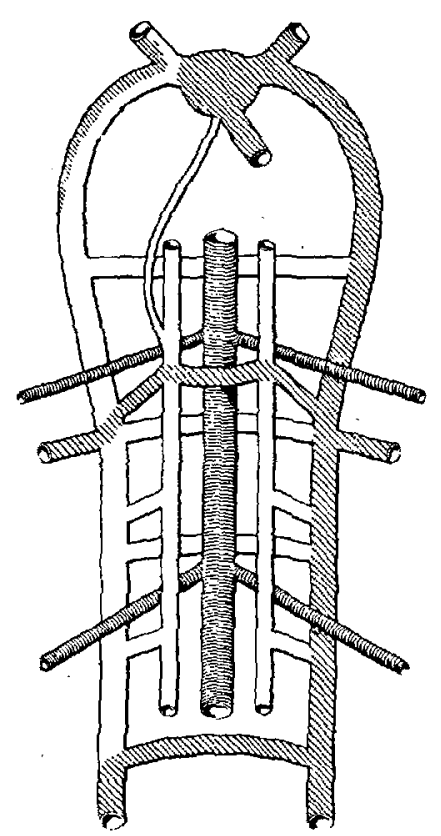

Fig. 9.

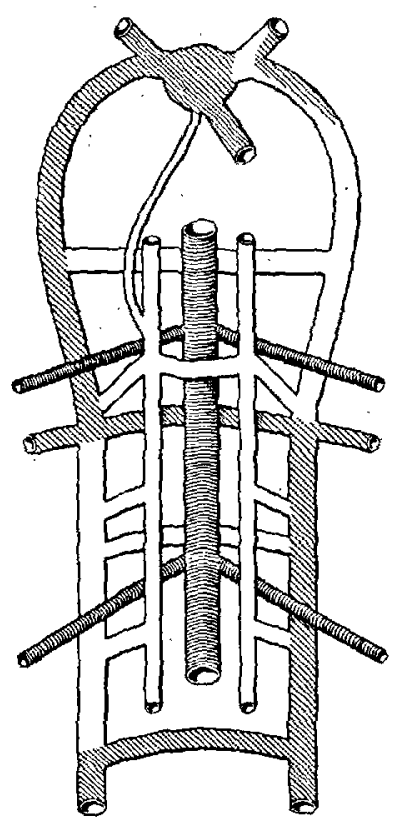

Fig. 10.

Variation A. 3. a. Fall von Neu- Variation A. 4. b. Fall von Hochborger.

stetter.

denn, dass man die Vena azygos als oberen Abschnitt der Cardinalvene betrachtet, wie in Fig. 9 zur Darstellung gebracht wurde. (Die Entstehungsweise der Venae spermaticae internae aus der Cardinalisschlinge ist hier ausser Betracht geblieben.)

A. 4. Beobachtungen, wo bei fehlender Vena cava inferior primitiva einmal ein Teil der rechten, anschliessend ein Teil

1) 19 . 
der linken Cardinalvene als Hauptgefäss auftritt, sind von $\mathrm{Dw}$ igh t ${ }^{1}$ ), Hochstetter ${ }^{2}$ ) und Koll $\mathrm{mann}^{3}$ ) beschrieben. In den drei Fällen bildet die linke Cardinalvene den unteren, die rechte den oberen Abschnitt der Abflussbahn. Im Grund-

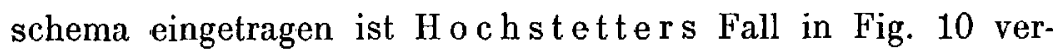
anschaulicht. Es handelt sich hier um einen dorsal von der Aorta verlaufenden Nierenblutabfluss, nach der Tabelle um einen Fall A.4.b.

Gruppe B. Die Vena cava inferior primitiva ist schwach entwickelt. Das Cardinalsystem befördert im wesentlichen das Blut der unteren Körperhälfte zum Herzen. Fälle dieser Art sind in der Literatur selten vertreten. Mir sind nur zwei derartige bekannt; eine von $\mathrm{Hans} \mathrm{Lauber}{ }^{4}$ ) beschriebene $\mathrm{Ab}$ weichung und der Fall, welcher zur vorliegenden Arbeit Veranlassung gab. Beide sind als zur Gruppe B. 4. b. gehörig zu betrachten, d. $h$. ein Teil der Hauptabflussbahn, und zwar in beiden Fällen der obere, ist als Endstück der rechten Cardinalvene zu deuten, während an diesem anschliessend ein Stück der linken Cardinalvene die Hauptabflussbahn bildet. Auch hierin stimmen die beiden Fälle überein, dass an der Lendenwirbelsäule beide Cardinales persistieren und dass die rechte Nierenvene, hinter der Aorta verlaufend, den linken Cardinalisstamm erreicht. Im Grundschema eingetragen, stellt Fig. 11 den in Fig. 1 abgebildeten Fall dar. Dieselbe schematische Figur könnte mit einigen unwesentlichen Änderungen auch für den Fall von $\mathrm{L}$ a u ber dienen.

Gruppe C. Die Vena cava inferior primitiva ist Hauptabflussbahn geworden, das Cardinalissystem befördert nur wenig Blut der unteren Körperhälfte zum Herzen.

Gruppie D. Die Vena cava inferior primitiva ist einzige $\mathrm{Ab}$ -

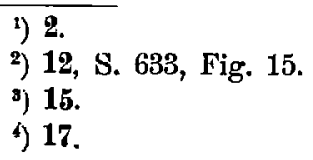


flussbahn, das Cardinalissystem befördert kein Blut der unteren Körperhälfte zum Herzen.

In die Gruppe $\mathrm{C}$ gehört bekanntlich der ,normale" Befund; nach der Tabelle entspricht dieser C. 2. a.; er ist im Grundschema eingetragen dargestellt in Fig. 5. Von Variationen gehören in diesen beiden Gruppen diejenigen Fälle, welche als Verdoppe-

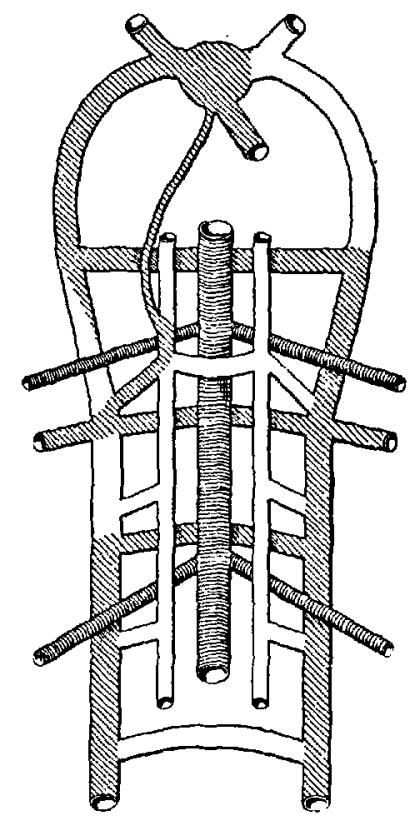

Fig. 11.

Variation B. 4. b. Sehema des Falles der Fig. 1.

lung oder Linkslagerung der unteren Hohlvene beschrieben worden sind. Betrachtet man die Venae azygos und hemiazygos als Endstücke der ursprünglichen Cardinalvenen, wie in unseren Figuren angenommen, so hängt die Entscheidung, ob ein bestimmter Fall zur Gruppe C: oder D gehört, davon ab, ob zwischen azygos resp. hemiazygos einerseits, den Nierenvenen oder den Venae lumbales ascendentes andererseits Verbindungen. vorhanden sind oder fehlen. Da dieser Punkt bei der Beschrei- 
bung der betreffenden abweichenden Zustände im allgemeinen wenig Berücksichtigung gefunden hat, so ist die Entscheidung oft nicht zu treffen.

Fälle von Verdoppelung der unteren Hohlvenen, welche in der Tabelle unter C. 1. oder D. 1. gehören, sind in grosser Anzahl beobachtet worden. $\mathrm{Z}$ ande $\mathrm{r}^{1}$ ) hat im Jahre 1892 nicht

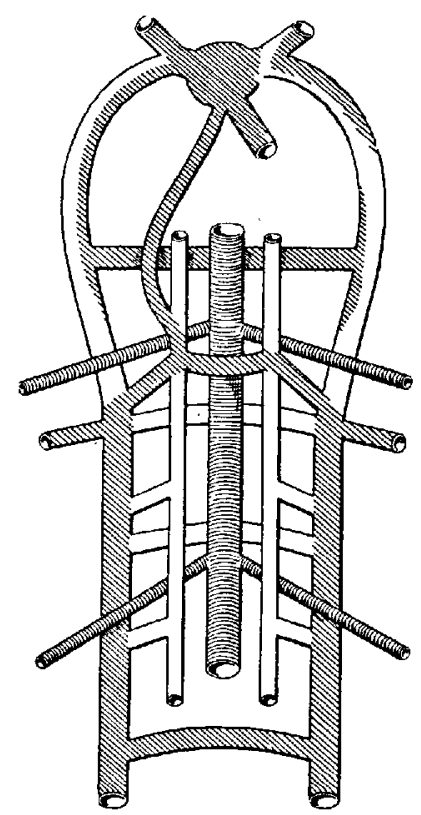

Fig. 12.

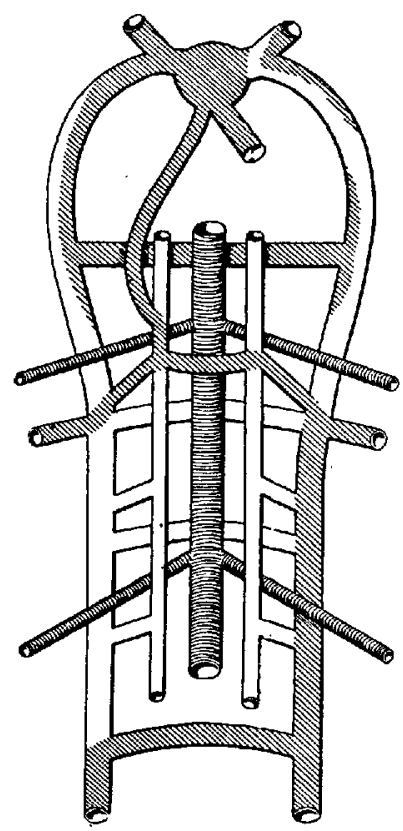

Fig. 13.

Variation C. 1. a. Fall von Risch. Variation C. 3. (D. 3). a. Fall von bieth. Fränkel.

weniger als 28 desbetreffende Beobachtungen zusammengestellt; seitdem sind noch einige beschrieben worden von Géra $\mathrm{rd}^{2}$ ), Kollmann ${ }^{3}$ ), Patten ${ }^{4}$ ), Rischbieth ${ }^{5}$ ).
1) Siehe 15.
) $5,6,8$.
3) 15 .
4) 21 .
5) 22. 
Von diesen zahlreichen Fällen sei hier der yom letztgenannten Autor beschriebene angeführt, er stimmt übrigens mit demjenigen von $\mathrm{Patten}$ fast genau überein. Es handelt sich nach der Tabelle um Fälle C. 1. a. Im Grundschema eingetragen sind sie in Fig. 12 dargestellt. Die Persistenz der beiden subrenalen Abschnitte der Venae cardinales kann verbunden sein mit einer mehr oder weniger stark entwickelten Vena iliaca transversa.

Als normal gilt, wie oben schon besprochen, der Zustand C. 2. a. (Fig. 5); jedoch kommt D. 2. a. vielleicht ebenso häufig vor. Der Zustand C. 2.b. resp. D. 2.b. (d. h. also: normaler Befund, ausser was den Verlauf der Vena renalis anbelangt, in dem ldiese hinter der Aorta verläuft) ist in der Literatur einige Male erwïhnt; so bei $\mathrm{Krause}$ in Henles Handbuch ${ }^{\mathbf{1}}$ ), in besonderen Aufsätzen von $A$. und L. Froriep ${ }^{2}$ ) und Gladston ${ }^{3}$ ). Es ist anzunehmen, dass in diesen Fällen eine der hinteren Anastomosen (s. Fig. 4) an iStelle der indirekten vorderen Verbindungen funktioniert ${ }^{4}$ ).

Der Zustand C.3. oder D. 3. (links liegende Vena cava inferior) ist beschrieben worden von $\operatorname{von~Alten}{ }^{5}$ ), Fränkel ${ }^{6}$ ) und Gérard ${ }^{7}$ ). Schematisiert ist Fränkels Beobachtung in Fig. 13 dargestellt. Alle drei Beobachtungen stimmen darin überein, dass die Queranastomose vor die Aorta verläuft; der interessante Fall, wobei eine hintere Anastomose sich vorfände, ist bisher meines Wissens nicht beobachtet worden.

1) 11, S. 369 .

2) 4 .

3) 9.

4) 4, S. 583 oben. Grosser berichtet; dass bei Mikrochiroptera die Vena renalis Sinistra normaliter dorsal von der Aorta verlaufe. 10, S. 355. 5) 1 .

9) 3.

7 . 7 . 
Ebensowenig sind als C.4. oder D.4. zu bezeichnende $\mathrm{Zu}$ stände angetroffen worden. És müsste in dem kurzen subrenalen Abschnitt ein Teil der rechten sowie der linken Cardinalvene als Hauptgefüss persistieren. Der Abstand scheint dafür zu kurz zu sein, was nicht ausschliesst, dass möglicherweise doch noch ein derartiger Befund gemacht werden könnte. 


\section{Literaturverzeichnis.}

Es ist nicht die Absicht, im unten folgenden Register alle bisher beschriebenen Variationen im besprochenen Gebiet anzufuhren. Als Fandgruben seien die Nummern 2, 12, 15 und 26 empfohlen, es finden sich dort Zusammenstellungen gleichartiger Fălle. Die mit einem * bezeichneten Nummern sind mir nicht zugrunglich gewesen.

1. 1913. Alten, Hans $v$, Uber linksseitige Lage der Vena cava inferior. Anat. Anz. Bd. 43. S. 337.

2. 1901. Dwight, T., Absence of the inferior cava below the diaphragm. Journ. of Anat. and Physiol. Vol. XXXV. S. 7.

3. 1910. Frinkel, Walter, Linksseitige Vena cava inferior. Anat. Anz. Bd. 37. S. 240.

4. 1895. Froriep, A. und L., Ober eine verhaltnismässig hulfige Varietat im Bereich der unteren Hohlvene. Anat. Anz. Bd. 10. S. 574.

5. 1903. Gérard, G., Duplicité apparente de la veine cave inférieure. Bibliographie Anatomique. Tome XII. S. 293.

6: 1906. Derselbe, Anomalies Vasculaires. Bibliographie Anatomique. Tome XV. S. 85.

7. 1908. Derselbe, Anomalie exceptionelle de la veine cave inférieure. Bibliographie Anatomique. Tome XVII. S. 227.

8. 1914. Derselbe, Duplicité apparente de la veine cave inférieure. Bibliographie Anatomique. Tome XXIV. S. 137.

9. 1911. Gladstone, R. J., Post-caval Ureter. Journ. of Anat. and Phys. Vol. XLV. S. 225.

10. 1901. Grossur, O., Zur Anatomie und Entwicklungsgeschichte des Gefăsssystems der Chiropteren. Anat: Hefte. Bd. XVII. S. 203.

11. 1868. Henle, J., Handbuch der systematischen Anatomie. Bd. 3.

12. 1898. Hoch stetter, F., Beitrihge zur Entwicklungsgeschichte des Venensystems der Amnioten, Morph. Juhrb. Bd. 20. S. 543.

13. 1908. Derselbe, Hertwigs Handbuch der Entwicklungslehre der Wirbeltiere. Bd. 3, 2. Téil.

14. 1913. Johnston, T. B., Anomaly of the vena cava inferior. Journ. of Anat, and Physiol. Vol. XLVII. S. 235.

15. 1893. Kollmann, J., Abnormitaten im Bereich der Vena cava inferior. Anat. Anz. Bd. 8. S. 97. 
16. 1911. Keibel und Mall, Entwicklungsgeschichte des Menschen. Bd. 2.

17. 1901. Laaber, Hans, Ein Fall von teilweiser Persistenz der hinteren Kardinalvenen beim Menschen. Anat. Anz. Bd. 19. S. 590.

18. 1901-02, Lewis, F. T., The development of the vena cava inferior. Amer. Journ, of Anat. Bd. 1. S. 229.

19. 1903. Neuberger, Hans, Ein Fall von vollkommener Persistenz der linken Vena cardinalis post. bei fehlender Vena cava inferior. Anat. Anz. Bd. 43. S. 65.

20. 1898. *Parker, G. H. and C. H. Tozier, The thoracic derivatives of the posteardinal veins in swine. Bull. Mus. Comp. Zool. Harvard.

21. 1909. Patten, C. J., Persistence of the embryonic Arrangements of the post-renal part of the cardinal veins. Anat. Anz. Bd. 34. S. 189.

22. 1914. Rischbieth, H., Anomaly of the inferior vena cava. Duplication of the post-renal segment. Journ. of Anat. and Physiol. Vol. XLVIII. S. 297.

43. 1914. Sabin, Flor. R., The development of the Azygos veins as shown in injected pig embryos. Proceedings Amer. Assoc. of Anatomists. 30. Session. Anat. Record. Vol. VIII. S. 82.

24. 1913. Waterston, D., Duplication of the postrenal segment of the cava inferior. Journ. of Anat. and Physiol. Vol. XLVII. S. 433.

25. 1892. * $Z$ ander, Über Verdoppelung der unteren Hohlvene. Verein $f$. wiss. Heilk. zu Königsberg i. P. Sitzıng vom 21. März 1892.

26. 1896. Zumstein, J, Zur Anatomio und Entwicklungsgeschichte des Menschen. Anat. Hefte. Bd. 6. S. 573.

27. 1897. Derselbe, Zur Entwicklung des Venensystems bei dem Meerschweinchen. Anat. Hefte. Bd. 8. S. 167. 